\title{
Verfahren der GIS-Modellierung von Erreichbarkeiten für Schlaganfallversorgungszentren
}

\author{
Sebastian Rauch ${ }^{1} \cdot$ Jürgen $\operatorname{Rauh}^{1}$
}

Eingegangen: 9. März 2016 / Angenommen: 30. August 2016 / Online publiziert: 19. September 2016

(C) Springer-Verlag Berlin Heidelberg 2016

Zusammenfassung Der methodisch orientierte Beitrag stellt ein Verfahren zur Modellierung von Versorgungsbereichen und Erreichbarkeiten von sogenannten Schlaganfallversorgungszentren (Stroke Units) in Deutschland mithilfe von Geographischen Informationssystemen (GIS) vor. Auf der Grundlage von OpenStreetMap-Vektordaten werden routingbasierte Einzugsgebiete der Schlaganfallversorgungszentren ermittelt und mit vektorbasierten Daten zur Demographie auf räumlich disaggregierter Ebene verschnitten. Eine Modellierung zeitlicher Erreichbarkeiten mit Rettungsfahrzeugen resultiert in Isochronen-Kartendarstellungen, welche in der Verschneidung mit hoch aufgelösten demographischen Sachdaten verschiedene Analysemöglichkeiten bieten. Diese werden an einem Fallbeispiel aus Unterfranken aufgezeigt, welches die Potenziale des Verfahrens für die raumbezogene Planung von Schlaganfallversorgungszentren skizzieren soll. Eine Anwendung des Verfahrens auf andere medizinische Einrichtungen oder die Verschneidung mit weiteren themenbezogenen Sachdaten ist möglich.

Die Arbeit beschreibt die methodische Grundlage für ein Kooperationsprojekt zwischen der Professur für Sozialgeographie und dem Institut für Klinische Epidemiologie und Biometrie der Universität Würzburg, in welchem ausführlich der Frage nach der Versorgungssicherheit von Schlaganfallpatienten nachgegangen wird.

Sebastian Rauch

sebastian.rauch@uni-wuerzburg.de

Prof. Dr. Jürgen Rauh

juergen.rauh@uni-wuerzburg.de

1 Institut für Geographie und Geologie, Julius-Maximilians-Universität Würzburg, Am Hubland, 97074 Würzburg, Deutschland
Schlüsselwörter Erreichbarkeitsanalyse · GIS · Gesundheitswesen · Schlaganfallversorgungszentren

\section{Method of GIS-based-modelling analyzing Accessibility for Stroke Units}

Abstract The methodologically-oriented paper presents a method for modeling supply areas and accessibility of stroke units in Germany with the use of geographic information systems (GIS). Based on OpenStreetMap-data vector routing based catchment areas of stroke units are analysed and blended with vector-based demographic data at a spatially disaggregated level. A modeling of temporal accessibility with rescue vehicles resulted in isochronemaps. The intersection of these maps with high-resolution demographic attributed data offers different analysis options. These are demonstrated in a case study from Lower Franconia, which is to outline the potential of the method for the spatial planning of stroke units. An application of the method to other medical institutions or the intersection with other thematic attribute data is possible.

Keywords Accessibility analysis · GIS · Healthcare · Stroke units

\section{Hintergrund und Fragestellung}

Im Zuge der demographischen Entwicklung kommt es insbesondere in ländlich peripheren Räumen zunehmend $\mathrm{zu}$ Schließungen oder Zusammenlegungen von Einrichtungen der Daseinsgrundvorsorge. Logische Konsequenz ist, dass die Entfernungen größer, die Erreichbarkeit schwieriger und die Transportkosten höher werden. Eine Ausdünnung des 
Angebots macht besonders kluge Standortentscheidungen, neue Angebotsformen und Erreichbarkeitsstrategien erforderlich, um die Bevölkerung in der Fläche angemessen zu versorgen (Kocks 2007).

Besondere Aufmerksamkeit erfährt in diesem Zusammenhang vor allem die medizinische Versorgung, da Vergrößerungen von Distanzen mit erhöhten Gefahren für die individuelle Gesundheit und im schlimmsten Falle mit einem erhöhten Sterbefallrisiko einhergehen können. Die öffentliche Diskussion um die Sicherstellung der stationären Versorgung hat zudem vor allem im Kontext der Einführung des Abrechnungsverfahrens nach Fallpauschalen (Diagnosis Related Groups Systems, DRG) in Deutschland (2005), verbunden mit der Abkehr vom Selbstkostendeckungsprinzip hin zur leistungsorientierten Vergütung, große Bedeutung erfahren (vgl. Malzahn/Wehner/Fahlenbrach 2011: 35). Malzahn/Wehner/Fahlenbrach (2011: 35) sehen in dem ,auftretenden Widerspruch zwischen staatlicher Angebotsplanung und wettbewerblicher Steuerung der Leistungserbringung“ gar eine „Legitimationskrise der Krankenhausplanung“ entstehen. Im Rahmen eines komplexer gewordenen Systems aus gesetzlichen Grundlagen und Akteurskonstellationen haben die an der Planung beteiligten Akteure und Behörden zwischen weitreichender Bedarfsdeckung und guter Erreichbarkeit für alle sozialen Gruppen und Regionen sowie Finanzierbarkeit abzuwägen. Bei der Erstellung der planerischen Grundlagen spielen auch methodische Aspekte eine wichtige Rolle, unter deren Anwendung Analysen mit dem Ziel durchgeführt werden können, Stärken und Schwächen der aktuellen Versorgungssituation aufzuzeigen und mögliche Optimierungsbedarfe $\mathrm{zu}$ identifizieren (vgl. Heuschmann/Busse/Wagner et al. 2010: 334).

Verkehrliche Erreichbarkeit ist ein Aspekt, dem in diesem Zusammenhang besondere Bedeutung zukommt. Es liegen inzwischen einige empirische Analysen wie modellhafte Berechnungen zur verkehrlichen Erreichbarkeit aufgrund der durch den demographischen Wandel zu erwartenden Standortveränderungen von Einrichtungen der (medizinischen) Daseinsvorsorge und Siedlungsmustern vor. Wie von Schweikart und Kistemann (2004) beschrieben, nehmen bei den Erreichbarkeitsstudien GIS-Analysen und kartographische Darstellungen eine inzwischen wichtige Rolle ein (vgl. Elliott/Wakefield/Best et al. 2001). In neueren Studien wird vor allem die Verfügbarkeit von Versorgungsinstitutionen durch derartige Methoden quantifiziert (vgl. Traub 2004; Neumeier 2013; Neumeier 2014; Seip/Bill/ Kinskofer 2015). Auch im Gesundheitswesen und speziell in der Krankenhausversorgung wurden in Deutschland Erreichbarkeitsstudien mittels Geographischer Informationssysteme durchgeführt. So stellte Kortevoß (2005) in seiner Dissertation unter anderem GIS-basierte Instrumente vor, mit denen zentrale Parameter der Krankenhausplanung wie die Entfernung zur Bevölkerung, Kapazitäten sowie Umfang der potenziell zu versorgenden Bevölkerung abgebildet werden können.

Hieran knüpft die vorliegende Studie an. Vorrangiges Ziel ist es, ein methodisches Verfahren und Vorgehen vorzustellen, die es erlauben, die räumliche Abdeckung von Schlaganfallversorgungszentren in Deutschland auf unterschiedlichen räumlichen Ebenen zu analysieren und zu bewerten. Wesentliches Anliegen ist es, ein möglichst realitätsnahes Erreichbarkeitsmodell mit GIS-Werkzeugen (tools) zu entwickeln, das

- die Entfernung in für die Notfallversorgung relevanteren Zeitdistanzen statt der in vielen Studien häufig verwendeten Luftlinien- oder Straßendistanzen misst,

- Routingalgorithmen verwendet, die auf einem digitalen Verkehrsnetz basieren,

- als niedrigste räumliche Bezugsebene Gebäude verwendet, denen Bevölkerungspotenziale zugeordnet werden.

Mithilfe des GIS-gestützten Modells kann beispielsweise das (hinsichtlich bevölkerungsstatistischer Merkmale differenzierbare) Bevölkerungspotenzial ermittelt werden, das von den jeweiligen Zentren in bestimmten Fahrzeitzonen versorgt wird. Weiterhin ermöglicht das Verfahren, Räume zu identifizieren, in welchen eine potenzielle Unterversorgung vorzufinden ist. Diese Analysen können somit als planerische Grundlagen für Optimierungspotenziale in der Schlaganfallversorgung dienen.

\section{Verkehrliche Erreichbarkeitsstudien}

Hinsichtlich des Begriffes „Erreichbarkeit“ unterscheiden Gather/Kagermeier/Lanzendorf (2008: 79) zum einen die Perspektive des Individuums oder Haushalts verbunden mit der Frage danach, „welche Gelegenheiten innerhalb einer bestimmten Wegezeit mit verschiedenen Verkehrsmitteln erreichbar sind“" (,personenbezogene Erreichbarkeit“, englisch access) und zum anderen die Perspektive eines potenziellen Zielortes verbunden mit der Frage, „wie viele Personen innerhalb bestimmter Wegezeiten oder mit einem beschränkten Kostenaufwand dorthin kommen können“" (Gather/Kagermeier/Lanzendorf 2008: 79) (,Erreichbarkeit“, „Einzugsbereich“, englisch accessibility). Diese scharfe begriffliche Trennung lässt sich in der fachlichen Diskussion oft nicht durchhalten (Gather/Kagermeier/ Lanzendorf 2008: 79) und unterliegt zumeist keinem einheitlichen Konzept (vgl. Neumeier 2014: 9). Im Kontext der Gesundheitsversorgung werden neben allgemeinen vor allem die beiden räumlichen Dimensionen des Zugangs availability (Verfügbarkeit) und accessibility (Entfernung) besonders hervorgehoben (vgl. Kortevoß 2005: 68 ff.). 
Auch die Operationalisierung von Erreichbarkeit variiert je nach Fragestellung (vgl. Gather/Kagermeier/Lanzendorf 2008: 81). Schwarze (2005: 9 f.) unterscheidet zwei Konzepte zum Messen von Erreichbarkeit in der Verkehrs- und Flächennutzungsplanung. Neben der ,einfachen Erreichbarkeit“", die ausschließlich auf die Güte des Verkehrssystems zielt und mit einfachen Erreichbarkeitsindikatoren gemessen werden kann, berücksichtigt die ,integrierte Erreichbarkeit" sowohl das Verkehrssystem als auch die Siedlungsstruktur. „Mathematisch formuliert ist die integrierte Erreichbarkeit [...] ein aus zwei Funktionen bestehendes Konstrukt, in dem die erste Funktion die von diesem Standort erreichbaren und realisierbaren Aktivitätsgelegenheiten repräsentiert und die zweite Funktion den Aufwand, der erforderlich ist, um die Aktivitätsgelegenheiten über das Verkehrssystem zu erreichen“" (Schwarze 2005: 10 f.).

Bei der Messung von räumlicher Erreichbarkeit im Gesundheitswesen differenziert Guagliardo (2004: 4 f.) vier verschiedene Kategorien von häufig verwendeten Maßzahlen, wobei er besonders neuere Entwicklungen zur Messung räumlicher Erreichbarkeit in der Kategorie der Gravitationsmodelle vorstellt. Diese Maßzahlen zur räumlichen Erreichbarkeit sowie Standort-Einzugsbereichs-Modelle haben in der praktischen Standort- und Bedarfsplanung eine lange Tradition und insbesondere durch die Entwicklung entsprechender GIS-Tools und dem Einsatz leistungsfähiger Computer auch Eingang und Erweiterungen in jüngere praktische Fragestellungen gefunden.

In der praktischen Anwendung wurde außerhalb des Gesundheitswesens unter anderem von Hollburg, Sinn und Voland (2012) eine Analyse zur Erreichbarkeit von touristischen Attraktionen durchgeführt. Auch bei der Validierung von Mobilitätssituationen im öffentlichen und privaten Verkehr werden derartige Untersuchungen häufig angewendet. Glander, Kramer und Döllner (2010) untersuchen beispielsweise die Mobilitätsqualität des Öffentlichen Personennahverkehrs (ÖPNV) in Berlin. Weitere exemplarische Studien zu Erreichbarkeiten wurden von Schwarze (2005) und Maier (2011) vorgenommen. Auf einer größeren Skala wird durch das European Observation Network (ESPON) im „Transport Accessibility at Regional/Local Scale and Patterns in Europe Report" (TRACC) die potenzielle Erreichbarkeit europäischer Regionen ermittelt. Raumzeitliche Informationen werden in dieser Studie mit weiteren Parametern wie zum Beispiel demographischen Daten verschnitten (Spiekermann/Wegener 2013). Vergleichbare Studien wurden zudem für den deutschen Raum durch Schürmann, Spiekermann und Wegener (2000) durchgeführt. Diese Erhebungen verdeutlichen die hohe Flexibilität der Datenkombinationen im Zuge einer Erreichbarkeitsanalyse und unterstreichen deren Einsatzmöglichkeiten in zahlreichen Kontexten. In der deutschen Raumforschung werden Erreichbarkeitsmodelle beispielsweise vom Bundesinstitut für
Bau-, Stadt- und Raumforschung (BBSR) durchgeführt, um Erreichbarkeiten von Autobahnen, von zentralen Orten und Agglomerationszentren im Bahn- und Pkw-Verkehr darzustellen. Diese Erreichbarkeitsmodelle verwenden $\mathrm{Nea}$ rest-neighbour- oder Nearest-center-Annahmen und werden in der Regel auf der Basis von Standorten und Verkehrsnetzen für Landkreise oder Gemeinden berechnet. Unter Verwendung von Nutzungshäufigkeiten und Einwohnerzahlen lassen sich damit auch Wege und Gesamtentfernungen feststellen. Der Nachteil vieler Erreichbarkeitsmodelle ist die Ausklammerung individuellen Mobilitätsverhaltens, das häufig mehrere Tätigkeitskopplungen auf einem Weg vorsieht und demnach von Nearest-center-Annahmen abweicht. Auch werden in einigen Modellen demographische Veränderungsprozesse nicht explizit berücksichtigt.

Im Gesundheitswesen wurden in Deutschland Erreichbarkeitsstudien insbesondere vor dem Hintergrund der Einführung der German-Diagnosis Related Groups (GDRGs) in den 2000er-Jahren durchgeführt. Ausführlich diskutiert Kortevoß (2005) unter anderem methodische Aspekte der Krankenhausplanung und wendet diese am Beispiel von Hessen u. a. am Beispiel von Stroke Units an. Zur Verortung der Bevölkerung wurde eine Aggregation auf sogenannte Marktzellen verwendet, was gegenüber noch kleinräumlicheren Zuordnungsverfahren Konsequenzen hinsichtlich der Genauigkeit der Entfernungsberechnungen, die in Kilometer-Angaben erfolgen, haben dürfte. Auch die Dissertation von Focke (2006) widmet sich der Krankenhausplanung, wobei ein Simulationsmodell erstellt wurde, das verschiedene Szenarien unter Variation mehrerer Einflussgrößen berechnen kann. In jüngerer Zeit rückt durch aktuelle Strukturreformen im somatischen Krankenhauswesen deren räumliche Versorgung weiter in den Vordergrund und wird zunehmend diskutiert (vgl. Scheller-Kreinsen/Krause 2015). Ein internationales Beispiel mit methodischem Schwerpunkt stammt von Jamtsho und Corner (2014). Sie behandeln die Erreichbarkeit primärer Gesundheitseinrichtungen in Bhutan unter Einsatz von GIS und unter Anwendung eines gravitationsbasierten Modells, das auch für Szenarien verwendet werden kann.

In den letzten Jahren fokussierten Studien zur Erreichbarkeit besonders auch auf die hausärztliche Versorgung vor allem in Räumen, in denen sich der demographische Wandel besonders stark bemerkbar macht. Berlin und Rulle (2009) untersuchen die Erreichbarkeit von Hausärzten in einem Landkreis in Mecklenburg-Vorpommern und prognostizierten eine Erhöhung der durchschnittlichen Fahrzeit von erheblichem Ausmaß in einigen Teilräumen des Uecker-Randow-Kreises. Werden in dieser Studie Praxisschließungen aufgrund von auf Zufallsauswahl beruhenden Annahmen modelliert, so hat Neff (2014) am Beispiel des Landkreises Schweinfurt (Bayern) ein empirisch gestütztes Simulationsverfahren entwickelt, mit dessen Hilfe Szena- 
rien $\mathrm{zu}$ unterschiedlichen Entwicklungen in der hausärztlichen Versorgung durchgeführt werden können. Sommer und Sauer (2012) analysieren die Erreichbarkeit von Hausärzten im Kyffhäuserkreis. Im Rahmen des EU-Projektes „European Rural Futures“ (EURUFU) befassen sie sich vor allem mit der Erreichbarkeit von Hausarztpraxen mit öffentlichen Verkehrsmitteln, thematisieren dabei aber auch den Einfluss des demographischen Wandels auf die Bevölkerungsstruktur (vgl. Sommer/Sauer 2012: 11).

\section{Schlaganfall und Schlaganfallversorgungs- zentren}

Schlaganfall, eine plötzlich auftretende schwere Durchblutungsstörung des Gehirns durch Hirninfarkt oder Hirnblutung, zählt zu den häufigsten neurologischen Erkrankungen (Kolominsky-Rabas/Heuschmann 2002: 658; AQUA 2015: 10). Heuschmann, Busse, Wagner et al. (2010: 334) berichten von über 196.000 erstmaligen und 66.000 wiederholten Schlaganfällen pro Jahr in Deutschland (Stand 2008), wobei die altersstandardisierten Neuerkrankungsraten bei Männern höher sind als bei Frauen. Jedoch erleiden mehr Frauen aufgrund der höheren Lebenserwartung einen Schlaganfall als Männer (Heuschmann/Busse/Wagner et al. 2010: 334). Dies drückt sich auch in den Todesfällen aus: Das Statistische Bundesamt (2016) führt Schlaganfall unter den häufigsten Todesursachen in Deutschland. Demnach verstarben 2014 10.621 Frauen und 6132 Männer an einem Schlaganfall; dies entspricht einem Anteil von 1,9\% (weiblich: 2,4; männlich: 1,5\%) an allen Todesursachen (Statistisches Bundesamt 2016: 4, 10). Dabei zeigt sich nicht nur eine deutliche Differenz zwischen den Geschlechtern, sondern auch hinsichtlich des Alters (vgl. Tab. 1). In den vergangenen Jahren gingen die Sterbefallzahlen mit der Todesursache Schlaganfall trotz demographischer Alterung stetig zurück (2002: 39.433; 2006: 28.566; 2010: 23.675; 2014: 16.753 (Statistisches Bundesamt 2004: o. S.; Statistisches Bundesamt 2007: 9; Statistisches Bundesamt 2012: 4; Statistisches Bundesamt 2016: 7)). Die Ursachen für diesen Rückgang sind sicher vielfältiger Art, allerdings darf unterstellt werden, dass der Aufbau von Stroke Units nicht unwesentlich mit dazu beigetragen hat.

Diese Stroke Units oder Schlaganfallversorgungseinrichtungen sind definiert als spezialisierte Versorgungszentren mit entsprechender Ausrüstung zur intensiven Betreuung und Überwachung von betroffenen Patienten (Hacke/ Schuster 1998: 520). „Entsprechend dem angelsächsischen Konzept sind Stroke Units kleine Spezialstationen für die ausschließliche Behandlung von Schlaganfallpatienten durch ein multiprofessionelles, auf die Schlaganfallbehandlung spezialisiertes Team. Das Behandlungskonzept umfasst sowohl die akute Behandlung von Schlaganfallpatienten als auch frühe Rehabilitationsanstrengungen“ (Ringelstein/Busse 2004: 7). Die ersten Stroke Units entstanden in den 1980er-Jahren in Skandinavien (Ringel-
Tab. 1 Sterbefälle 2014 mit Todesursache Schlaganfall (nicht als Blutung oder Infarkt bezeichnet) nach Altersgruppen und Geschlecht

\begin{tabular}{lccc}
\hline Altersgruppen & Männlich & Weiblich & Gesamt \\
\hline Unter 1 Jahr & - & - & - \\
1 bis unter 5 Jahre & - & - & - \\
5 bis unter 10 Jahre & - & - & - \\
10 bis unter 15 Jahre & - & - & 1 \\
15 bis unter 20 Jahre & - & 1 & 2 \\
20 bis unter 25 Jahre & - & 2 & 2 \\
25 bis unter 30 Jahre & 1 & 1 & 3 \\
30 bis unter 35 Jahre & 2 & 1 & 7 \\
35 bis unter 40 Jahre & 5 & 2 & 10 \\
40 bis unter 45 Jahre & 7 & 3 & 79 \\
45 bis unter 50 Jahre & 18 & 7 & 132 \\
50 bis unter 55 Jahre & 60 & 19 & 257 \\
55 bis unter 60 Jahre & 92 & 40 & 446 \\
60 bis unter 65 Jahre & 182 & 75 & 987 \\
65 bis unter 70 Jahre & 309 & 137 & 2133 \\
70 bis unter 75 Jahre & 612 & 375 & 3340 \\
75 bis unter 80 Jahre & 1122 & 1011 & 4548 \\
85 bis unter 85 Jahre & 1397 & 1943 & 4781 \\
90 Jahre und älter & 1406 & 3142 & 16.753 \\
Summe & 919 & 3862 &
\end{tabular}

Quelle: Statistisches Bundesamt (2016: 7) 
Abb. 1 Konzeption der Erreichbarkeitsanalyse

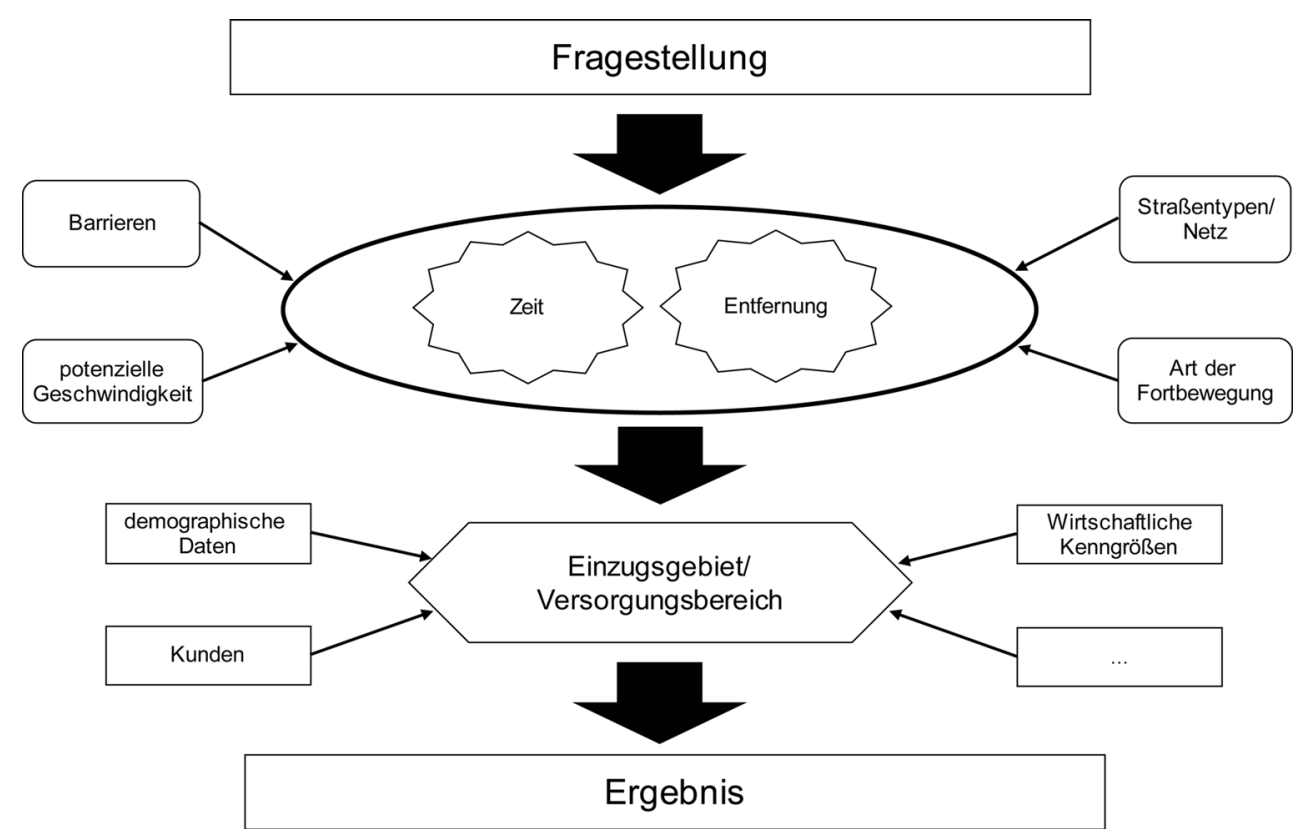

stein/Busse 2004: 7), seit Mitte der 1990er-Jahre werden derartige Zentren verstärkt in Deutschland eingerichtet (Heuschmann/Busse/Wagner et al. 2010: 336). Stroke Units werden in Deutschland durch die Deutsche SchlaganfallGesellschaft (DSG) aufgrund von Kriterien in den Bereichen „strukturelle Voraussetzungen“, „Diagnostik und Monitoring“, ,personelle Ausstattung“ und „Prozesse und Standards“ zertifiziert (DSG 2012). Zum Zeitpunkt der Analyse erfüllten 279 Schlaganfallversorgungseinrichtungen in Deutschland die notwendigen Kriterien (DSG 2015).

\section{Konzeptionierung der Erreichbarkeitsanalyse}

Die Analyse und Simulation der Erreichbarkeit von Versorgungseinrichtungen erlaubt es, auf der Basis unterschiedlicher Indikatoren und Parameter Strukturmaßnahmen für bestehende und zukünftige Institutionen zu entwickeln (vgl. Krafft/König/Kortevoß 2004: 224). Hierzu zählen Optimierungsprozesse von Fahrtrouten, aber auch beispielsweise Standortanpassungen bestimmter Einrichtungen. Die Entfernung ist dabei stets ein wichtiges Produkt des aktuellen Verkehrssystems (vgl. Schürmann/Spiekermann/Wegener 1997; Spiekermann/Wegener 2012: 9; Neumeier 2014: 9). Über ein der Analyse zugrundeliegendes Netzwerk aus Kanten und Knoten kann letztendlich die Reichweite einer oder mehrerer Einrichtungen bestimmt werden. Abb. 1 zeigt, welche Faktoren in einer Erreichbarkeitsanalyse eine Rolle spielen.

In einem netzwerkbasierten System stellen die Zeit und die Entfernung die wichtigsten Variablen dar. Während die Zeit als Einflussgröße die Fortbewegungsgeschwindigkeit im bestehenden Netzwerk angibt, ergibt sich die Entfernung aus den potenziell zur Verfügung stehenden Kanten des Systems und deren Länge. Aufgrund der Übertragbarkeit und des in der Realität größeren Anwendungsbereiches soll folglich nicht von den häufig stark generalisierten Luftliniendistanzen ausgegangen werden, sondern stets von routingabhängigen Pfaden. Diese Pfade werden durch die im Untersuchungsraum gegebenen Verbindungen durch StraBen und Wege definiert. Die Dimensionen einer Erreichbarkeitsanalyse können je nach Untersuchungsgegenstand sehr vielfältig sein. Aus diesen Dimensionen, wie beispielsweise die Fortbewegungsart oder Barrieren, lassen sich Indikatoren für den potenziellen Versorgungsbereich ableiten (vgl. Spiekermann/Wegener 2012: 11). Die daraus erzeugten, standortabhängigen Einzugs- bzw. Versorgungsbereiche ermöglichen eine Kombination mit räumlich erfassbaren Parametern wie Einwohnerzahl sowie weiteren demographischen und sozioökonomischen Merkmalen. Je nach Forschungsfrage und Gegenstand lassen sich daraus örtliche Potenziale ableiten.

\section{Datengrundlage und Methodik}

Als Routinggrundlage für die Analyse der Erreichbarkeit von Stroke Units werden die frei verfügbaren Vektordaten von OpenStreetMap (OSM) verwendet. Vorteil dieser Daten ist es, dass sie flächendeckend in einer hohen Auflösung für ganz Deutschland erhältlich sind. Ihre Eignung für derartige Projekte wurde durch Seip, Bill und Kinskofer (2015) belegt und zeigt sich zudem auch in der zunehmenden Verwendung für routingbasierende Fragestel- 
Tab. 2 Geschwindigkeiten nach Straßenklassen

\begin{tabular}{lc}
\hline Straßenklasse & $\begin{array}{l}\text { Angenommene Geschwindig- } \\
\text { keit (in km/h) }\end{array}$ \\
\hline Fußgängerzone & 25 \\
Verkehrsberuhigter Bereich, Feldweg, Kreisverkehr & 30 \\
Notfallzufahrt, Notfallbucht, Zubringerstraße & 40 \\
Kreuzung, Schnellstraßenabfahrt, Bundesstraßenzufahrt, Landstraßen- & 45 \\
zufahrt, Hauptstraßenzufahrt & 60 \\
Ortsstraße, Nebenstraßen & 95 \\
Landstraße, Bundesstraße & 115 \\
Schnellstraße, Autobahn &
\end{tabular}

Quelle: Nach Pütz (2005) und in Absprache mit Rettungssanitätern (Fahrten mit Sondersignalen)
Tab. 3 Vergleich Vektor- vs. Rasterdatenmodell

\begin{tabular}{lll}
\hline & Vorteile & Nachteile \\
\hline Vektorbasierte & Geringerer Datenaufwand & Keine interne Differenzierung der \\
Erreichbarkeit & & Isochronen \\
& Schnelle Berechnung & Statische Isochrone \\
& Klare Einzugsgebiete & Geringe Flexibilität \\
Rasterbasierte & Stufenlos, diskrete Raumdarstellung & Benötigt gute Netzabdeckung \\
Erreichbarkeit & Leichte Verknüpfungsmöglichkeiten & Hoher Rechenaufwand \\
& Gute Differenzierungsmöglichkeiten & Hohe Komplexität \\
& & Einzugsgebiete in hohem Maße auflö- \\
& & sungsabhängig \\
& & Keine klare Einzugsbereiche \\
& & Stark auflösungsabhängig \\
\hline
\end{tabular}

Quelle: Eigene Darstellung nach Neumeier (2014) lungen (vgl. Hollburg/Sinn/Voland 2012; Kucharska/Pieper/ Schweikart 2014).

Mithilfe von ArcGIS werden die Vektordaten nach deren Aufarbeitung in ein routingfähiges Netzwerk, bestehend aus Kanten und Knoten, überführt. Die anschließend durchgeführten Routingberechnungen basieren auf euklidischen Distanzmodellen, welche bei ausreichender Verkehrsnetzdichte, wie im mitteleuropäischen Raum nahezu vollständig vorzufinden, exakte Ergebnisse erzeugen (vgl. Dahlgren 2008: 16). Als wichtige Modellvariable für die folgende Analyse muss jeder Straßentyp mit einer potenziellen Geschwindigkeit versehen werden. Die Werte variieren in Abhängigkeit des Straßentyps und wurden in Anlehnung an das Erreichbarkeitsmodell des BBSR (Pütz 2005) sowie in Absprache mit Rettungssanitätern aus medizinischen Versorgungseinrichtungen festgelegt (vgl. Tab. 2). ${ }^{1}$ Der Datensatz wurde um nicht relevante Straßentypen (unter anderem Reitwege, Fähren) bereinigt. Eine differenzierte Geschwindigkeitsbetrachtung von Straßen inner- und außerorts wurde im Zuge dieser Analyse nicht durchgeführt, sodass die Geschwindigkeitsannahmen im Verlaufe eines Pfades konstant bleiben. Barrieren, welche im normalen Straßenverkehr auftreten, wie beispielsweise maximale Geschwindigkeiten oder

${ }^{1}$ Die Absprache erfolgte über den Kooperationspartner Institut für Klinische Epidemiologie und Biometrie der Universität Würzburg.
Ampelkreuzungen, wurden aufgrund der durch den $\S 35$ Straßenverkehrsordnung geltenden Sonderrechte der Einsatzfahrzeuge nicht in die Modelldarstellung aufgenommen (vgl. Bayerisches Rotes Kreuz 2008). Des Weiteren wurden vereinfachend weder tageszeitabhängige, noch wochentagsabhängige Verkehrssituationen unterschieden.

Als Ausgangspunkte dienen adressbasierte Informationen zu den Schlaganfallversorgungszentren. Die Standortinformationen basieren auf Daten der Deutschen Schlaganfall-Gesellschaft (DSG 2015). Um im späteren Verlauf der Analyse feststellen zu können, wie viele Personen potenziell versorgt werden, wurden die Bevölkerungszahlen jeder Ortschaft des Zensus 2011 (Punktdaten) und die Grundflächen sämtlicher Gebäude der Ortschaften aus den OSMDaten (Polygone) verwendet. Die Einwohnerzahlen wurden bewusst als Punktdaten gewählt, da diese Herangehensweise die am höchsten aufgelöste, von administrativen Gebietszuweisungen unabhängige Datenquelle darstellt.

\subsection{Versorgungsbereichsanalyse}

Nach der Überführung der OSM-Straßendaten in ein routingfähiges Netzwerk können diese mithilfe der Tools der ArcGIS - Extension Network Analyst verarbeitet werden. Für die folgende Untersuchung wurde das Tool Service Area verwendet, welches ausgehend von den gewählten Versor- 


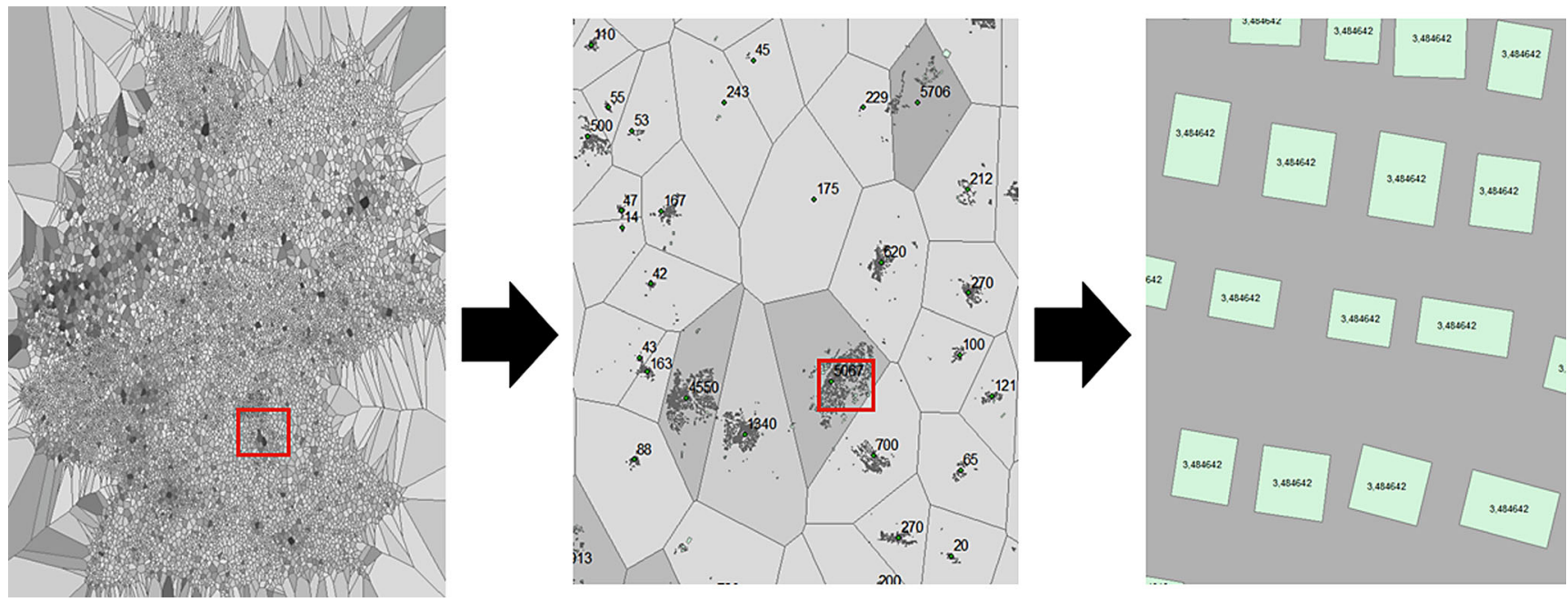

Abb. 2 Disaggregation der Bevölkerungsinformationen

gungszentren Polygone bzw. Isochrone der jeweiligen Versorgungsbereiche erzeugt.

Die Größe der Polygone hängt von den gesetzten Grenzwerten der Isochronen ab und kann über die Grenzen des Untersuchungsgebietes hinausreichen. Da es sich bei einem Schlaganfall um ein Notfallereignis handelt, welches zur Reduzierung des Risikos von Folgeschäden schnellstmöglich behandelt werden muss, ist der bestimmende Faktor die Zeit. Um einen ersten Einblick in die Abdeckung der untersuchten Schlaganfallversorgungszentren zu erhalten, wurden als maximale Versorgungszeit 30 min festgelegt und zur besseren Interpretation der Ergebnisse diese Gesamtzeit in 10-Minuten-Isochrone unterteilt. Die 30-Minuten-Grenze wurde auf gemeinsame Initiative des Institutes für Notfallmedizin und Medizinmanagement, des Klinikums der Universität München sowie der Arbeitsgemeinschaft der Südwestdeutschen Notärzte veranschlagt (vgl. Bundesärztekammer 2007).

Der vektorbasierte Ansatz eignet sich aufgrund der klar definierten Grenzen und der erheblichen Datenmenge im zu untersuchenden Raum. In der Praxis existiert neben diesem Verfahren noch der rasterbasierte Lösungsansatz. Beide weisen fallspezifische Vor- und Nachteile für eine Erreichbarkeitsanalyse auf, welche in Tab. 3 aufgeführt sind.

Für beide Modelltypen gilt: Die Güte der Erreichbarkeitsanalyse ist im hohen Maße von der Güte des zugrundeliegenden navigationsfähigen Netzwerks abhängig. Rasterbasierte Daten ermöglichen es, Ergebnisse der Modellierung leicht mit weiteren Daten zu verknüpfen. Abgesehen von dem sehr hohen Rechen- und Speicheraufwand dieser Methode gehen auch klare Einzugsgebietsgrenzen verloren. In gering aufgelösten Rasterdaten zeigen sich weniger starke Verschneidungsprobleme mit Sekundärdaten, jedoch werden vor allem bei räumlich hochaufgelösten Daten jene stark aggregiert. Eine ausführliche Darstellung eines sol- chen Ansatzes wird durch Schürmann, Spiekermann und Wegener (1997) gegeben. Über ein indirektes Verfahren, welches im Folgenden dargestellt wird, ist es auch im vektorbasierten Ansatz möglich, weitere Daten mit den erzeugten Polygonen zu verschneiden.

\subsection{Vektorbasierte Verschneidung der Isochronen mit Bevölkerungsdaten}

Zunächst müssen die in Zentroiden vorliegenden Bevölkerungsdaten auf die zu untersuchenden flächenhaften Polygone disaggregiert werden. Um ein von administrativen Grenzen unabhängiges Modell zu erzeugen, wurde auf der Basis der Zensusdaten ein Netz aus Thiessen-Polygonen für das gesamte Bundesgebiet erzeugt ${ }^{2}$. Auf diese Weise wird auch der Einfluss der außerhalb des Untersuchungsgebietes liegenden Gemeinden erfasst. Die sich daraus ergebende Bevölkerungsverteilung dient nun als Grundlage für die Berechnung der Bevölkerungspotenziale in den jeweiligen Einzugsbereichen der Schlaganfallversorgungszentren. Um eine möglichst hohe Auflösung der Analyse gewährleisten zu können, werden sämtliche, im jeweiligen Untersuchungsgebiet liegenden Gebäudepolygone mit den entsprechenden Bevölkerungsinformationen verschnitten. Ziel ist es, jedem einzelnen Gebäudepolygon eine modellierte Personenzahl zuzuweisen, auf deren Basis dann eine Gesamtzahl in einem definierten Raumabschnitt bestimmt werden kann. Hierzu wird die Bevölkerungszahl des zugrundeliegenden Thiessen-Polygons durch die Anzahl der in jenen

\footnotetext{
2 Als Thiessen-Polygone werden jene Flächen bezeichnet, welche durch das Thiessen-Polygon-Verfahren oder Voronoi-Polygon-Verfahren erzeugt werden. Sie ergeben sich aus der Verbindung der Mittelsenkrechten der Verbindungslinie zweier Punkte mit kürzester Distanz (vgl. De Lange 2006: 360 f.).
} 
befindlichen Gebäudegrundflächen geteilt. Die Anzahl der potenziell durch das jeweilige Schlaganfallversorgungszentrum versorgten Personen ergibt sich dann aus den kumulierten Bevölkerungszahlen der in den Isochronen befindlichen Gebäudepolygone (Vektordaten). Aufgrund der klaren Isochronengrenzen und den räumlich hochaufgelösten Bevölkerungsinformationen erweist sich der zuvor beschriebene Vektoransatz als gut geeignet. Zudem eignet sich für diese Fragestellung die verwendete Disaggregationsmethodik mithilfe der Gebäudegrundflächen in besonderem Maße (vgl. Abb. 2). Die Gebäudegrundflächen umfassen alle Gebäudetypen (z. B. Wohngebäude, Arbeitsstätten, Dienstleis- tungseinrichtungen). Da sich Personen nicht nur in Wohngebäuden aufhalten (und dort einen Schlaganfall erleiden können), erschien es sinnvoll, sämtliche Gebäudetypen in die Modellierung mit einzubeziehen. Da darüber hinaus keine Informationen zur Wohnfläche oder zum Gebäudeaufriss zur Verfügung standen, wurden die Personen einer Ortschaft gleichmäßig über die Gebäude verteilt. Dies ist zwar nicht ganz unproblematisch, denn Bevölkerungskonzentrationen (z. B. in Hochhäusern) oder kaum von Menschen genutzte Gebäude werden dabei nicht differenziert. Diese Unterscheidung wäre bei kleinräumigeren Analysen durchaus realisierbar, für eine großräumige Analyse wäre
Abb. 3 Zeitliche Erreichbarkeit von zertifizierten Stroke Units in Unterfranken

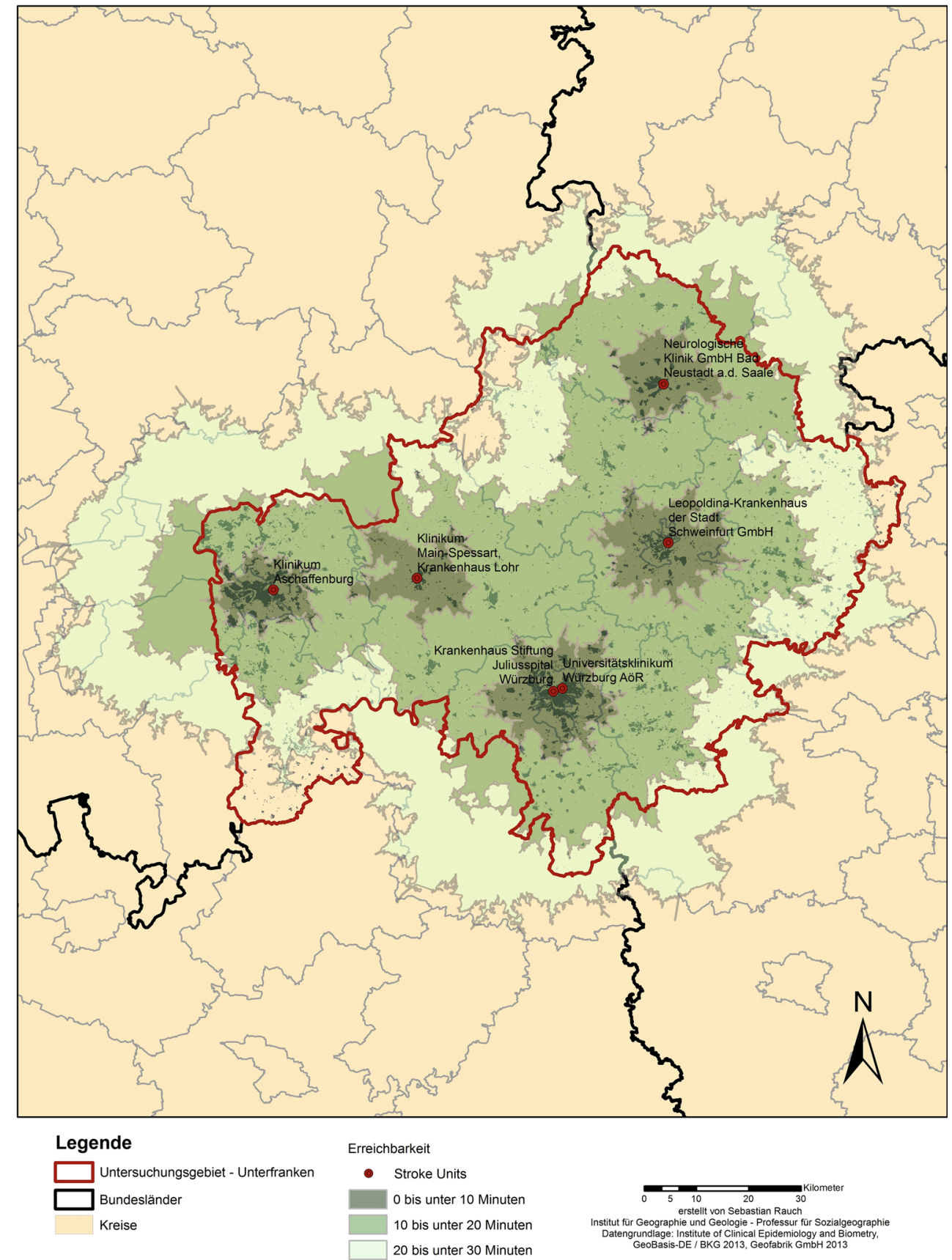


der Datenbeschaffungs- und Rechenaufwand nicht vertretbar gewesen.

\section{Analysemöglichkeiten und ausgewählte Teilergebnisse}

Auf der Basis der ermittelten Isochronen und der modellierten Bevölkerungsverteilung ergeben sich verschiedene Analysemöglichkeiten auf unterschiedlichen räumlichen Ebenen, wovon einige im Folgenden fallbeispielhaft aufgezeigt werden. Neben deutschlandweiten Betrachtungen

Tab. 4 Durch zertifizierte Stroke Units in Unterfranken potenziell Versorgte nach Entfernungszonen

\begin{tabular}{lrcl}
\hline & $\begin{array}{l}\text { Potenziell } \\
\text { versorgte } \\
\text { Personen }\end{array}$ & $\begin{array}{l}\text { Anteil dis- } \\
\text { junkt } \\
\text { (in \%) }\end{array}$ & $\begin{array}{l}\text { Anteil kumu- } \\
\text { liert } \\
\text { (in \%) }\end{array}$ \\
\hline 0-10 min & 595.143 & 46,04 & 46,04 \\
$10-20 \mathrm{~min}$ & 561.937 & 43,48 & 89,52 \\
$20-30 \mathrm{~min}$ & 122.364 & 9,48 & 99,00 \\
Über 30 min & 12.983 & 1,00 & 100,00 \\
Gesamt & 1.292 .427 & 100,00 & - \\
\hline
\end{tabular}

können insbesondere Erreichbarkeiten und Versorgungsgrad für einzelne Regionen wie auch für einzelne Schlaganfallversorgungszentren analysiert werden.

Zunächst kann die Anzahl der potenziell versorgten Personen für Deutschland oder für ausgewählte Teilräume nach Entfernungszonen ermittelt werden. Bei der Betrachtung der individuellen Schlaganfallversorgungszentren können, um alle potenziellen Personen zu erfassen, Mehrfachzählungen auftreten. Die räumlichen Überschneidungen resultieren aus der Nähe der Versorgungseinrichtungen zueinander. Unter Annahme einer maximal 30-minütigen Erreichbarkeit der derzeit 279 in Deutschland durch die Deutsche Schlaganfall-Gesellschaft zertifizierten Schlaganfallversorgungszentren (Stand: 10.10.2015; DSG 2015) werden knapp $87 \%$ der Fläche Deutschlands abgedeckt. Erwartungsgemäß liegt eine hohe Konzentration der Zentren in den Agglomerationsräumen und größeren Städten vor. Unter Zugrundelegung der gewählten Parameter ist in diesen Städten und ihrem Umland eine lückenlose Versorgung gegeben. In Teilräumen Mecklenburg-Vorpommerns, Sachsen-Anhalts, Brandenburgs aber auch Bayerns sind die Distanzen zwischen den Einrichtungen größer bzw. die Abdeckung weniger dicht. Aus diesem Grund sind
Tab. 5 Altersverteilung der potenziell versorgten Personen nach Isochronen der Stroke Units in Unterfranken

\begin{tabular}{llll}
\hline Alter & Bis 10 min & 10 bis 20 min & 20 bis 30 min \\
\hline Unter 3 Jahre & 17.943 & 11.938 & 486 \\
3 bis unter 6 Jahre & 19.392 & 12.903 & 526 \\
6 bis unter 10 Jahre & 28.535 & 18.986 & 774 \\
10 bis unter 15 Jahre & 40.848 & 27.179 & 1107 \\
15 bis unter 18 Jahre & 27.576 & 18.348 & 748 \\
18 bis unter 20 Jahre & 19.186 & 12.766 & 520 \\
20 bis unter 25 Jahre & 48.218 & 32.083 & 1307 \\
25 bis unter 30 Jahre & 45.695 & 30.403 & 1239 \\
30 bis unter 35 Jahre & 41.458 & 27.584 & 1124 \\
35 bis unter 40 Jahre & 50.129 & 33.354 & 1359 \\
40 bis unter 45 Jahre & 65.634 & 43.671 & 1779 \\
45 bis unter 50 Jahre & 66.201 & 44.048 & 1795 \\
50 bis unter 55 Jahre & 56.371 & 37.507 & 1528 \\
55 bis unter 60 Jahre & 50.331 & 33.488 & 1365 \\
60 bis unter 65 Jahre & 37.287 & 24.809 & 1011 \\
65 bis unter 75 Jahre & 84.471 & 56.204 & 2290 \\
75 Jahre und älter & 67.122 & 44.660 & 1820 \\
\hline
\end{tabular}

Quelle: Eigene Berechnung nach Zensus 2011

Tab. 6 Potenzielle Versorgtenzahlen der zertifizierten Stroke Units in Unterfranken

\begin{tabular}{|c|c|c|c|c|c|c|}
\hline & $\begin{array}{l}\text { Uniklinikum } \\
\text { Würzburg }\end{array}$ & $\begin{array}{l}\text { Juliusspital } \\
\text { Würzburg }\end{array}$ & $\begin{array}{l}\text { Leopoldina } \\
\text { Krankenhaus } \\
\text { Schweinfurt }\end{array}$ & $\begin{array}{l}\text { Neurologische } \\
\text { Klinik Bad Neu- } \\
\text { stadt }\end{array}$ & $\begin{array}{l}\text { Klinikum } \\
\text { Main-Spes- } \\
\text { sart Lohr }\end{array}$ & $\begin{array}{l}\text { Klinik Aschaffen- } \\
\text { burg }\end{array}$ \\
\hline Bis $10 \mathrm{~min}$ & 207.529 & 213.401 & 135.147 & 43.923 & 36.096 & 167.265 \\
\hline 10 bis $20 \mathrm{~min}$ & 221.088 & 225.974 & 188.596 & 118.109 & 142.461 & 376.624 \\
\hline 20 bis $30 \mathrm{~min}$ & 375.461 & 371.922 & 480.118 & 298.938 & 647.688 & 1.414 .030 \\
\hline Gesamt & 804.077 & 811.297 & 803.862 & 460.970 & 826.245 & 1.957 .919 \\
\hline
\end{tabular}


Abb. 4 Zeitliche Erreichbarkeit zertifizierter Stroke Units in Unterfranken einschließlich Landkreise aus benachbarten Bundesländern

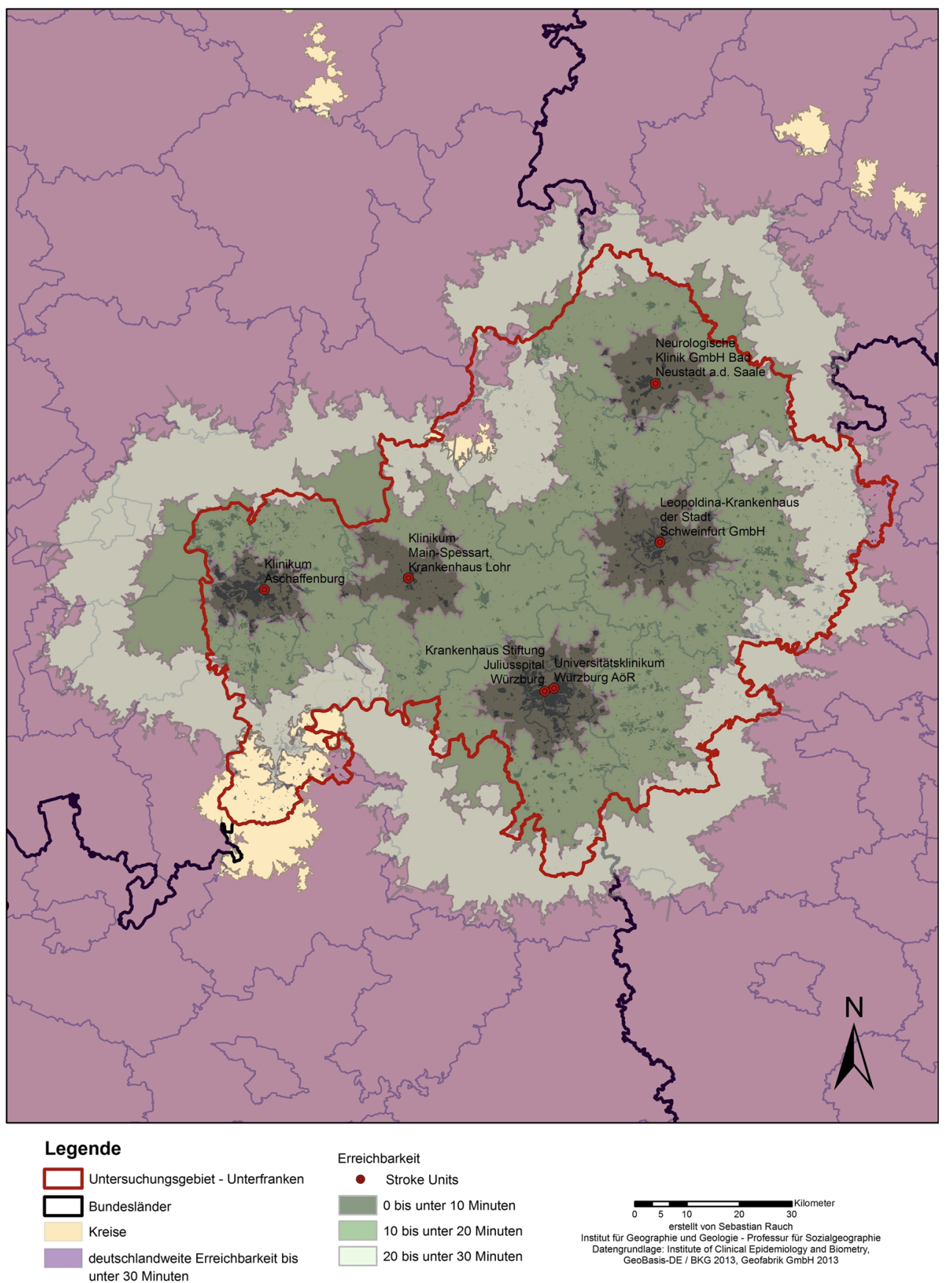

vor allem in Nordostdeutschland gemäß der angelegten Definition große Bereiche unterversorgt. Vor allem der Landkreis Stendal und der Altmarkkreis Salzwedel, aber auch der Landkreis Ludwigslust-Parchim weisen große unterversorgte Bereiche auf. Etwa 94,4\% der dort ansässigen Bevölkerung kann nicht binnen 30 min ein Schlaganfallversorgungszentrum erreichen. Des Weiteren befinden sich in Nordthüringen, vor allem im Landkreis Nordhausen und dem Unstrut-Hainich-Kreis, ferner dem Kyffhäuserkreis Defizitärräume. Nach dem beschriebenen Vorgehen betrifft dies in Deutschland etwa 2.804.000 Menschen.
Eine detaillierte Betrachtung auf regionaler Ebene soll exemplarisch für Unterfranken in Bayern mit sechs zertifizierten Stroke Units erfolgen (vgl. Abb. 3). Die Standorte befinden sich in Würzburg (2), Aschaffenburg, Schweinfurt, Bad Neustadt und Lohr am Main. Von den 1.292.427 Einwohnern in Unterfranken können 1.279.443 Menschen (99,0\%) von diesen sechs Standorten gemäß dem Modell binnen 30 min erreicht werden. Die verbleibenden knapp 13.000 Einwohner (1\%) außerhalb der 30-minütigen Erreichbarkeitszone konzentrieren sich auf Teilräume der Landkreise Miltenberg und Bad Kissingen. Eine weitere 
Abb. 5 Zeitliche Erreichbarkeit von zertifizierten Stroke Units und telemedizinischen Einrichtungen in Unterfranken

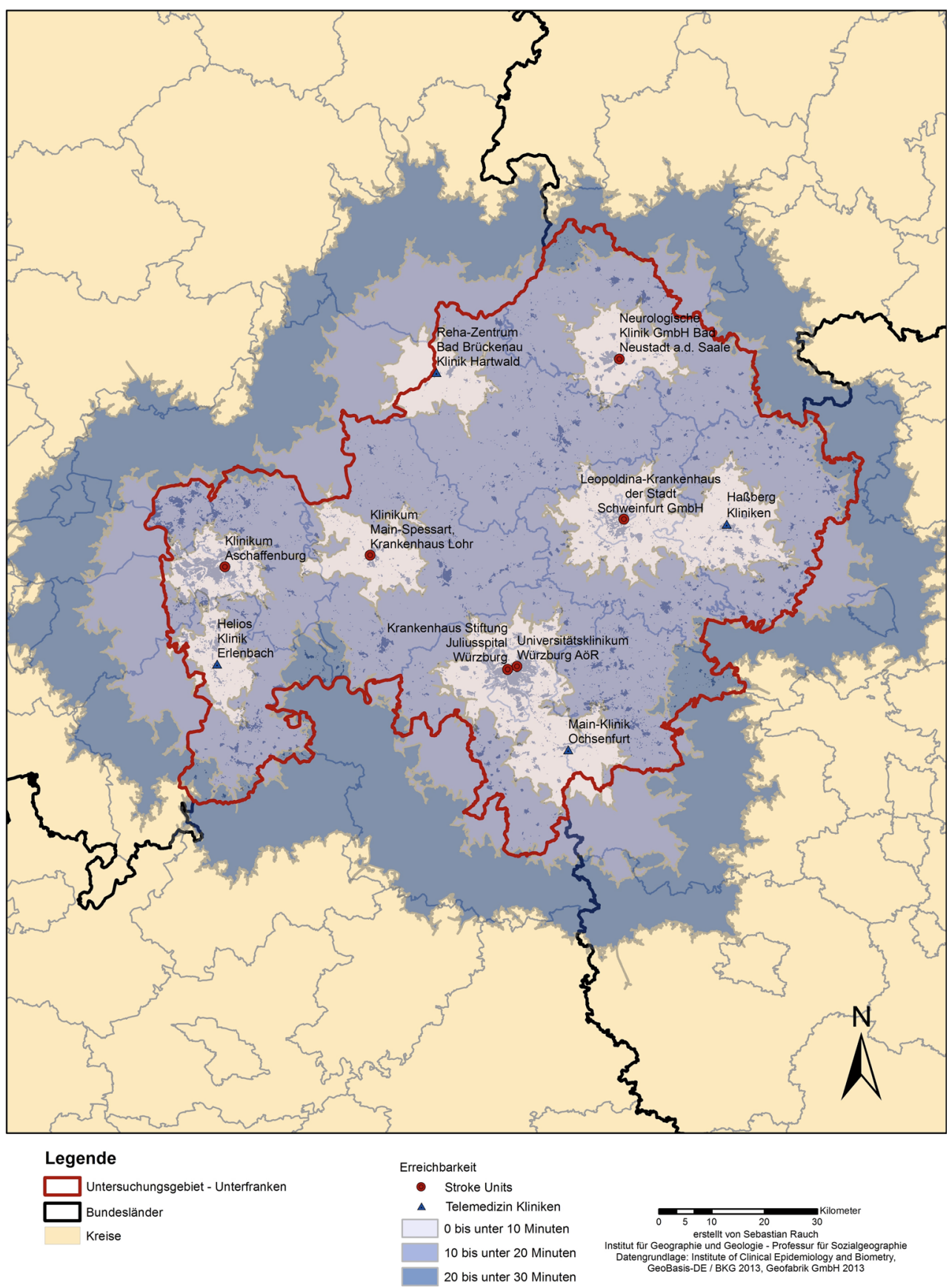

Differenzierung lässt sich nach Entfernungszonen durchführen (vgl. Tab. 4).

Durch die in Unterfranken befindlichen Schlaganfallversorgungseinrichtungen können potenziell rund $46 \%$ der Einwohner Unterfrankens in mindestens 10 min Fahrzeit erreicht werden. Weitere rund 560.000 Personen $(43,5 \%)$ sind binnen der nächsten 10 min zu erreichen.

Wenn in die Betrachtung auch die umliegenden Stroke Units benachbarter Bundesländer (Hessen, Thüringen, Baden-Württemberg) sowie der bayerischen Regierungsbezirke Mittel- und Oberfranken einbezogen werden, verändert sich die Situation der unterversorgten Räume nur im nördlichen Landkreis Bad Kissingen, während in den betroffenen Teilräumen des Landkreises Miltenberg keine Verbesserung der Erreichbarkeiten ermittelt werden (vgl. Abb. 4).

Die vorgestellte Methodik ermöglicht es, über das gleiche Verfahren der Disaggregation den Gebäudepolygonen Daten, den Personen demographische Merkmale wie Geschlecht oder Alter zuzuweisen. Zu diesem Zweck können demographische Daten aus dem Zensus 2011 mit den Isochronen verschnitten werden. Tab. 5 zeigt die Altersvertei- 
Abb. 6 Vergleich der zeitlichen Erreichbarkeit von zertifizierten Stroke Units sowie von Stroke Units und telemedizinischen Einrichtungen in Unterfranken

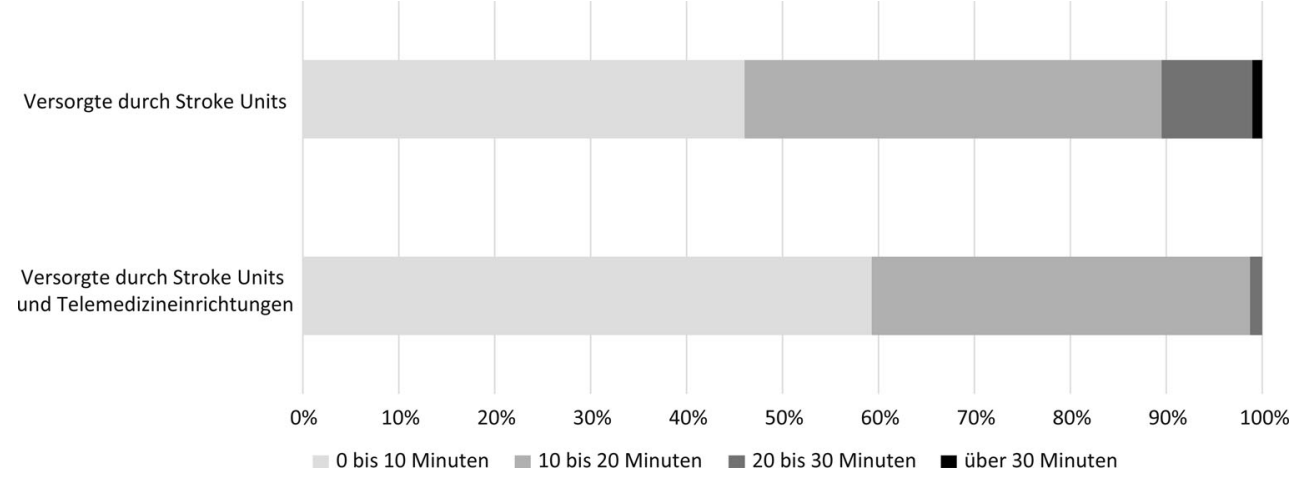

lung der potenziell versorgten Personen innerhalb Unterfrankens (2011) nach den jeweiligen Isochronen.

Eine Analyse der Altersstruktur und der durch einen Schlaganfall potenziell Betroffenen ist sinnvoll, da die Wahrscheinlichkeit des Eintretens eines solchen Ereignisses grundsätzlich mit dem Alter zunimmt. Mit einem Alter von 65 Jahren und älter, somit der statistisch gesehen am stärksten gefährdeten Personengruppe, werden etwa 256.500 Menschen im Untersuchungsgebiet versorgt. Von den knapp 13.000 Personen, die in einer Entfernungszone bis 30 min nicht von einer Stroke Unit erreicht werden, sind etwa $22 \% 65$ Jahre oder älter.

Die Analysen lassen sich auch für einzelne Schlaganfallversorgungszentren durchführen (vgl. Tab. 6). Da sich die Isochronen überschneiden können, können Personen im Versorgungsbereich mehrerer Stroke Units liegen. Um für jede einzelne Versorgungseinrichtung alle potenziell Betroffenen ermitteln zu können, wurden jene Personen dementsprechend mehr als einmal erfasst. Da situationsabhängig entschieden wird, welche Stroke Unit angefahren wird, ist diese Herangehensweise zur Untersuchung einzelner Kliniken für das Beispiel sinnvoll. Der Typ bzw. die Schwere des Schlaganfalls sowie die Ausstattung der jeweiligen Stroke Units sind hierbei die ausschlaggebenden Parameter der Wegeentscheidung. In der Analyse werden auch Versorgungsbereiche außerhalb Unterfrankens miteinbezogen. So ergeben sich für die Einrichtung der Klinik Aschaffenburg aufgrund der Lage im Verdichtungsraum Frankfurt/Main relativ hohe potenzielle Versorgtenzahlen.

Das GIS-Modell gestattet auch das Durchspielen von geplanten oder alternativen Angebots- und Versorgungsformen und deren Erreichbarkeiten. So existieren in Unterfranken vier Telemedizin-Einrichtungen des TRANSIT-StrokeTelemedizinnetzwerkes (vgl. Kraft/Kleinschnitz/Wiedmann et al. o.J.). Diese Einrichtungen verfügen zwar über keine zertifizierten Stroke Units, haben allerdings die Fähigkeit zur Versorgung von Schlaganfallpatienten. Werden diese Einrichtungen an der Main-Klinik Ochsenfurt, Helios Klinik Erlenbach, an den Haßberg-Kliniken in Haßfurt sowie an der Klinik Bad Brückenau in die Erreichbarkeitsanaly- se mitaufgenommen, verbessert sich die Versorgungslage in Unterfranken. Insbesondere tragen die Kliniken in Erlenbach und Bad Brückenau dazu bei, dass die vorher in der 30-Minuten-Isochrone nicht versorgten Bereiche jetzt abgedeckt werden (vgl. Abb. 5). Aber nicht nur in der Entfernungszone jenseits der $30 \mathrm{~min}$, sondern auch in den näheren Bereichen, würden sich durch zusätzliche Telemedizineinrichtungen die Erreichbarkeiten für viele potenzielle Patienten verkürzen (vgl. Abb. 6).

\section{Fazit und Ausblick}

Am Beispiel der Schlaganfallversorgung wurde gezeigt, wie routingbasierte Einzugsgebiete mit vektorbasierten Daten gekoppelt werden können. Vektordaten von OpenStreetMap (OSM) wurden dazu mit ArcGIS in ein routingfähiges Netz überführt. Unter Anwendung von straßentypenspezifischen Geschwindigkeiten von Rettungsfahrzeugen wurde mit euklidischen Distanzmodellen eine Modellierung der zeitlichen Erreichbarkeit von Schlaganfallversorgungszentren (Stroke Units) möglich. Dazu wurde das Instrument der Tools der ArcGIS - Extension Network Analyst verwendet, mit dem sich Isochronen der Versorgungsbereiche generieren lassen. Um eine möglichst hohe Auflösung der Analyse zu erreichen, wurden die Sachdaten zur Demographie aus dem Zensus 2011, die auf Ortsebene als Punktdaten vorliegen, im GIS-Modell auf einzelne Gebäudepolygone disaggregiert. Sowohl die eindeutigen, aus der Praxis vorgegebenen Grenzen der Isochronen als auch die differenzierten demographischen Sachdaten ermöglichen mit diesem Vektoransatz vielfältige Analysemöglichkeiten. Diese können auf unterschiedlichen räumlichen Ebenen durchgeführt werden und geben Hinweise auf Potenziale oder Defizite, was wiederum für die zukünftige Versorgungsplanung von Bedeutung sein kann.

Durch Ermitteln von Erreichbarkeitspotenzialen ergeben sich zahlreiche Möglichkeiten, komplexe räumliche Gegebenheiten $\mathrm{zu}$ analysieren. Künftig können unter anderem Reaktionszeiten der Notfalldienste in die Berechnung ein- 
gehen, um Hilfs- und Eintrefffristen evaluieren zu können. Auch kann der analysierte Bestand der 279 deutschen Stroke Units beliebig um weitere schlaganfallversorgende Einrichtungen wie die 148 Kliniken mit Neurochirurgie in Deutschland erweitert werden. Die gewählte Methodik erlaubt des Weiteren bevölkerungsspezifische Indikatoren in eine Erreichbarkeitsanalyse einzubeziehen. Hierzu zählen nicht nur demographische Daten, sondern auch sozioökonomische Indikatoren wie beispielsweise Einkommen, Bildung oder Pkw-Besitz. Dies ermöglicht die Übertragbarkeit des Verfahrens auch auf andere Anwendungsbereiche. Für das gewählte Beispiel eines Schlaganfalls, dessen Eintrittswahrscheinlichkeit mit dem Alter zunimmt, lassen sich Resultate durch Datenkombination weiter präzisieren. Das Ausweisen von Risikobereichen stellt hierbei ebenso eine Möglichkeit dar wie die Risikoprognose über Verknüpfungen mit regionsspezifischen Bevölkerungsvorausberechnungen.

Das vorgestellte Modell bildet weiterhin eine Grundlage für „Location-Allocation-Modellierungen“ (vgl. Drezner/ Hamacher 2004), mit deren Hilfe neben den Standortzuordnungen auch insbesondere in unterversorgten Räumen Standortbestimmungen potenziell neuer Schlaganfallversorgungszentren erfolgen können.

Ein weiteres Einsatzgebiet des vorgestellten Erreichbarkeitsmodells ergibt sich für die ambulante medizinische Versorgung. Anders als beim vorgestellten Beispiel der zentralen Schlaganfallversorgung in Unterfranken führt vor allem das demographisch bedingte Ausdünnen von Standorten (vor allem Arztpraxen) zu immer längeren Wegen und einer Steigerung des Verkehrsaufwandes. Dabei ließen sich Alternativangebote beispielsweise in Form von E-healthMaßnahmen als Szenarien unter Bedingungen demographischer Veränderungen in Erreichbarkeitsmodelle integrieren.

\section{Literatur}

AQUA - Institut für angewandte Qualitätsförderung und Forschung im Gesundheitswesen (2015): Versorgungsqualität bei Schlaganfall. Konzeptskizze für ein Qualitätssicherungsverfahren. https://www. g-ba.de/downloads/39-261-2283/2015-06-18_AQUA_AbnahmeKonzeptskizze-Schlaganfall.pdf (19.07.2016).

Bayerisches Rotes Kreuz (2008): Blaulichtbelehrung Fahrzeugeinweisung. http://www.bildung.brk.de/bildungsangebote-und-termine/ rettungsdienst/blaulichtbelehrung.pdf (19.07.2016).

Berlin, C.; Rulle, M. (2009): Demographischer Wandel und Daseinsgrundfunktionen. Erreichbarkeit von Hausärzten im Uecker-Randow-Kreis, Mecklenburg-Vorpommern. In: Geographische Rundschau $61,7 / 8,54-60$.

Bundesärztekammer (2007): Eckpunkte Notfallmedizinische Versorgung der Bevölkerung in Klinik und Präklinik. http:// www.bundesaerztekammer.de/fileadmin/user_upload/downloads/ Eckpunkte_Med_Notfallversorgung.pdf (19.07.2016).

Dahlgren, A. (2008): Geographic Accessibility Analysis. Methods and Application. Dissertation am Lund Institute of Technology.

De Lange, N. (2006): Geoinformatik in Theorie und Praxis. Berlin.
Drezner, Z.; Hamacher, H. W. (Hrsg.) (2004): Facility location. Applications and theory. Berlin.

DSG - Deutsche Schlaganfall-Gesellschaft (2012): Zertifizierungskriterien 2012. http://www.dsg-info.de/images/stories/DSG/PDF/ StrokeUnits/Aktualisierte\%20Zertifizierungskriterien_08_2012. pdf (19.07.2016).

DSG - Deutsche Schlaganfall-Gesellschaft (2015): Zertifizierte Stroke Units. http://www.dsg-info.de/stroke-units/stroke-units-uebersicht. html (27.07.2016).

Elliott, P.; Wakefield, J.; Best, N.; Briggs, D. (Hrsg.) (2001): Spatial Epidemiology: Methods and Applications. Oxford.

Focke, A. (2006): Regionale Leistungs- und Krankenhausplanung. Ein Simulationsmodell auf Basis eines Ameisenalgorithmus. Dissertation an der Universität Duisburg-Essen.

Gather, M.; Kagermeier, A.; Lanzendorf, M. (2008): Geographische Mobilitäts- und Verkehrsforschung. Berlin, Stuttgart.

Glander, T.; Kramer, M.; Döllner, J. (2010): Erreichbarkeitskarten zur Visualisierung der Mobilitätsqualität im ÖPNV. In: Kartographische Nachrichten 60, 3, 137-142.

Guagliardo, M. F. (2004): Spatial accessibility of primary care: concepts, methods and challenges. In: International Journal of Health Geographics 3, 3, 1-13.

Hacke, W.; Schuster, H.-P. (1998): Schlaganfallstationen (Stroke Units) - Zankapfel zwischen Internisten und Neurologen oder gemeinsame Aufgabe? In: Intensivmedizin und Notfallmedizin $35,7,519-522$.

Heuschmann, P. U.; Busse, O.; Wagner, M.; Endres, M.; Villringer, A.; Röther, J.; Kolominsky-Rabas, P.L.; Berger, K. (2010): Schlaganfallhäufigkeit und Versorgung von Schlaganfallpatienten in Deutschland. In: Aktuelle Neurologie 37, 333-340.

Hollburg, H.; Sinn, C.; Voland, P. (2012): Hier bin ich - Was kann ich erreichen? Webbasierte, interaktive Erreichbarkeitsanalyse touristischer Ziele der Stadt Potsdam, In: Strobl, J.; Blaschke, T.; Griesebner, G. (Hrsg.): Angewandte Geoinformatik 2012. Beiträge zum 24. AGIT-Symposium Salzburg. Berlin, 317-322.

Jamtsho, S.; Corner, R. J. (2014): Evaluation of spatial accessibility to primary healthcare using GIS. In: Annals of the Photogrammetry, Remote Sensing and Spatial Information Sciences 2, 2, 79-86.

Kocks, M. (2007): Konsequenzen des demographischen Wandels für die Infrastruktur im ländlichen Raum. In: Geographische Rundschau 59, 2, 24-31.

Kolominsky-Rabas, P. L.; Heuschmann, P. U. (2002): Inzidenz, Ätiologie und Langzeitprognose des Schlaganfalls. In: Fortschritte der Neurologie Psychiatrie 70, 12, 657-662.

Kortevoß, A. (2005): Krankenhausplanung unter Bedingungen der German-Diagnosis Related Groups. Methodischer Beitrag zur Ausgestaltung ordnungspolitischer Handlungsoptionen der Bundesländer. Dissertation an der Ludwig-Maximilians-Universität München.

Krafft, T.; König, A.; Kortevoß, A. (2004): Optimierung der Versorgung chronisch Kranker - GIS-basierte Analyse eines Disease Managment-Programms (DMP) für Diabetes. In: Schweikart, J.; Kistemann, T. (Hrsg.): Geoinformationssysteme im Gesundheitswesen. Grundlagen und Anwendung. Berlin, Offenbach, 211-224.

Kraft, P.; Kleinschnitz, C.; Wiedmann, S.; Heuschmann, P.U.; Volkmann, J. (o. J.): Transregionales Netzwerk für Schlaganfallintervention mit Telemedizin (TRANSIT-Stroke). https://www.transitstroke.de/pdf/Artikel_TRANSIT-Stroke.pdf (19.07.2016).

Kucharska, W.; Pieper, J.; Schweikart, J. (2014): Zugang zur Kindergesundheit in Brandenburg - eine Untersuchung auf Grundlage freier Geodaten. In: Strobl, J.; Blaschke, T.; Griesebner, G.; Zagel, B. (Hrsg.): Angewandte Geoinformatik 2014, Beiträge zum 26. AGIT-Symposium Salzburg. Berlin, Offenbach, 282-291.

Maier, D. (2011): Routing und Visualisierung im individuellen ÖPNV. In: Strobl, J.; Blaschke, T.; Griesebner, G. (Hrsg.): Angewandte Geoinformatik 2011. Berlin, Offenbach, 387-392. 
Malzahn, J.; Wehner, C.; Fahlenbrach, C. (2011): Krankenhausplanung in der Krise? Zum Stand und zur Weiterentwicklung der Sicherstellung der stationären Versorgung. In: Jacobs, K.; Schulze, S. (Hrsg.): Sicherstellung der Gesundheitsversorgung. Neue Konzepte für Stadt und Land. Berlin, 35-72.

Neff, C. (2014): Individuenbasierte Multiagentensimulation des Zusammenspiels von demographischem Wandel und hausärztlicher Versorgung im ländlichen Raum. Untersuchung am Beispiel des Landkreises Schweinfurt. Mannheim. = Berichte des Zentrums für Regionalforschung 4.

Neumeier, S. (2013): Modellierung der Erreichbarkeit öffentlicher Apotheken. Untersuchung zum regionalen Versorgungsgrad mit Dienstleistungen der Grundversorgung. Braunschweig. = Thünen Working Paper 14.

Neumeier, S. (2014): Modellierung der Erreichbarkeit von Supermärkten und Discountern. Untersuchung zum regionalen Versorgungsgrad mit Dienstleistungen der Grundversorgung. Braunschweig. $=$ Thünen Working Paper 16 .

Pütz, T. (2005): Erreichbarkeitsmodell des BBSR. http://www.bbsr. bund.de/BBSR/DE/Raumbeobachtung/UeberRaumbeobachtung/ Komponenten/Erreichbarkeitsmodell/erreichbarkeitsmodell_node. html (19.07.2016).

Ringelstein, E. B.; Busse, O. (2004): Stroke Units in Deutschland - Gefährdung eines Erfolgsrezeptes? In: Gesundheit und Gesellschaft: das AOK-Forum für Politik, Praxis und Wissenschaft 4, 3, 7-13.

Scheller-Kreinsen, D.; Krause, F. (2015): Die Ausgangslage für eine Strukturbereinigung: Fahrzeiten, Krankenhauserreichbarkeit und -kapazitäten. In: Klauber, J.; Geraedts, M.; Friedrich, J.; Wasem, J. (Hrsg.): Krankenhaus-Report 2015: Schwerpunkt: Strukturwandel. Stuttgart, 23-40.

Schürmann, C.; Spiekermann, K.; Wegener, M. (1997): Accessibility Indicators. Model and Report. SASI Deliverable D5. Dortmund. = Berichte aus dem Institut für Raumplanung 39.

Schürmann, C.; Spiekermann, K.; Wegener, M. (2000): Erreichbarkeit und Raumentwicklung. In: Deiters, J.; Gräf, P.; Löffler, G. (Hrsg.): Nationalatlas Bundesrepublik Deutschland, Band 9: Verkehr und Kommunikation. Heidelberg, 124-127.
Schwarze, B. (2005): Erreichbarkeitsindikatoren in der Nahverkehrsplanung. Dortmund. = Institut für Raumplanung, Arbeitspapier 184.

Schweikart, J.; Kistemann, T. (2004): Geoinformation in der Gesundheit - Entwicklung und Potenziale. In: Schweikart, J.; Kistemann, T. (Hrsg.): Geoinformationssysteme im Gesundheitswesen. Grundlagen und Anwendungen. Berlin, Offenbach, 3-14.

Seip, C.; Bill, R.; Kinskofer, A. (2015): Erreichbarkeitsanalysen auf Grundlage von Open-Streetmap und ArcGIS Online zur Notfallversorgung in Mecklenburg-Vorpommern. In: gis.Science 3, 94-104.

Sommer, S.; Sauer, T. (2012): Erreichbarkeit von Hausärzten im Kyffhäuserkreis. http://www.serviceagentur-demografie.de/fileadmin/ medien/dokumente/projekte/EU-Projekte/Bericht_hausaerzteeurufu_29102012-v4.PDF (19.07.2016).

Spiekermann, K.; Wegener, M. (2012): Dimensionen der Erreichbarkeit - von global bis lokal. In: Raumplanung 165, 6, 8-14.

Spiekermann, K.; Wegener, M. (2013): TRACC Transport Accessibility at Regional/Local Scale and Patterns in Europe Applied Research 2013/1/10 Final Report I Version 06/02/2015 Volume 2 TRACC Scientific Report. Dortmund.

Statistisches Bundesamt (2004): Gesundheitswesen. Todesursachen in Deutschland 2002. Wiesbaden. $=$ Fachserie 12 Reihe 4.

Statistisches Bundesamt (2007): Todesursachen in Deutschland. Gestorbene in Deutschland an ausgewählten Todesursachen 2006. Wiesbaden. $=$ Fachserie 12 Reihe 4 .

Statistisches Bundesamt (2012): Gesundheit. Todesursachen in Deutschland 2010. Wiesbaden. $=$ Fachserie 12 Reihe 4.

Statistisches Bundesamt (2016): Gesundheit. Todesursachen in Deutschland 2014. Wiesbaden. $=$ Fachserie 12 Reihe 4.

Traub, K.-P. (2004): Die Untersuchung der räumlichen Verbreitung von Gesundheitseinrichtungen mit GIS am Beispiel Hamburg. In: Schweikart, J.; Kistemann, T. (Hrsg.): Geoinformationssysteme im Gesundheitswesen. Grundlagen und Anwendung. Berlin, Offenbach, 241-256. 